\section{Sports injury}

\section{LACROSSE-RELATED INJURIES AMONG BOYS AND GIRLS TREATED IN US EMERGENCY DEPARTMENTS, 2000-2016}

Rebecca McAdams, Joseph Banojo, Kristin Roberts, Jingzhen Yang, Lara McKenzie. Nationwide Children's Hospital

\subsection{6/injuryprev-2020-savir.66}

Purpose Lacrosse is one of the fastest growing sports in the United States (US). Research is limited, and there are no known studies utilizing a nationally representative data set to analyze lacrosse injury patterns in the youth population over several years. This study sought to describe the epidemiology of lacrosse-related injuries treated in United States (US) hospital emergency departments (EDs).

Methods/Approach We conducted retrospective analysis using the National Electronic Injury Surveillance System data for youth aged 11-18 years who were treated for lacrosse-related injuries in US EDs from 2000-2016. Based on review of the case narratives, we created and coded a new injury mechanism variable. We generated national estimates from 6,408 cases.

Results An estimated 206274 lacrosse-related injuries to youths aged 11-18 years were treated in US EDs from 20002016. The rate of injuries per 10,000 youths significantly increased from 1.9 in 2000 to 5.3 in 2012 ( $p<0.0001)$, followed by significantly decreasing to 3.4 in $2016(p=0.020)$. Injury mechanism, body region injured, and diagnosis differed by sex. Boys were 1.62 times (95\% CI: 1.25-2.09) as likely to be injured by player-to-player contact. Girls were 2.21 times (95\% CI: 1.96-2.49) as likely to have non-contact injuries. Patients were divided into different age groups: 11-12, $13-14,15-16$, and 17-18. As age increased, the percentage of injuries from lacrosse sticks decreased and player-to-player contact increased.

Conclusions Despite additional protective regulations in the sport, injuries still occur and differ by the sex and age of the athlete. This study supports the continuation, modification, and addition of rules aimed to reduce lacrosse injury risk.

Significant and contribution to the field Understanding lacrosserelated injuries is critical as lacrosse-related injuries are a significant source of pediatric injury and ED utilization.

\section{Motor vehicle crashes: epidemiology and interventions}

\section{RANDOMIZED TRIAL OF SOCIAL COMPARISON FEEDBACK AND FINANCIAL INCENTIVE STRATEGIES FOR REDUCING HANDHELD PHONE USE WHILE DRIVING IN A NATIONAL USAGE-BASED AUTO INSURANCE PROGRAM}

M Kit Delgado. University of Pennsylvania, Penn Injury Science Center

10.1136/injuryprev-2020-savir.67

Statement of Purpose We conducted a randomized trial (\#NCT03833219) of behavioral interventions to reduce handheld phone use while driving among 2,108 customers from 42 states in a usage-based insurance program (Progressive
Insurance Snapshot Mobile). We hypothesized that behaviorally designed incentives would be more effective than a standard insurance incentive.

Methods/Approach Participants were randomized to 6 arms in a 50-day intervention period: (1) control (usual care); (2) social comparison feedback; (3) standard end of rating period incentive ( $\max \$ 50)$; (4) standard end of rating period incentive (max \$50) + social comparison feedback; (5) weekly, loss framed incentive $(\max \$ 50)+$ social comparison feedback; and (6) weekly, loss framed incentive (max \$100) + social comparison feedback. We used fractional regression to compare handheld phone use (seconds/hour of driving) measured by the Snapshot Mobile app between intervention arms versus the usual care arm.

Results Compared with usual care, neither social comparison feedback alone, or a standard incentive $(\max \$ 50)$ alone lead to a statistically significant reduction in phone use. However, when the standard incentive was paired with social comparison feedback, handheld phone use was reduced by -31 (95\% CI: - 74 to -13) seconds/hour of driving. The group assigned to a weekly, loss-framed incentive (max $\$ 50)$ paired with social comparison feedback experienced the largest reduction in handheld phone (-63 [95\% CI: -95 to -31 ] seconds/hour of driving).

Conclusions In a national usage-based auto insurance program, augmenting standard financial incentives with weekly feedback comparing a driver's phone use to other customers, and redesigning incentives to be delivered more frequently and to leverage loss-aversion reduced phone use while driving.

Significance and Contributions to Injury and Violence Prevention Science Given that American drivers average 300 hours of driving per year, behaviorally designed incentives for reducing phone use while driving have major potential for public health impact.

\section{Domestic and sexual violence}

\section{AMPLIFYING DAY-TO-DAY SEXUAL VIOLENCE HEALING EXPERIENCES THROUGH PHOTOGRAPHY AND STORYTELLING}

Laura Sinko. University of Pennsylvania

\subsection{6/injuryprev-2020-savir.68}

Purpose The purpose of this work is to discuss the therapeutic and translational value of using creative research activities to describe the healing journeys of survivors of campus sexual violence.

Approach Ethnographic narrative interviews $(n=24)$ and follow up photo-elicitation interviews $(n=19)$ were conducted with survivors of undergraduate sexual violence to help them describe their healing journeys. These stories were translated into a video and an interactive photography exhibit built with community partners to build a group narrative about what healing after sexual violence looks and feels like.

Results Overall, participants noted the therapeutic value of engaging in this type of research and that the visual nature of the work gave them new ways of looking at their situation. Two hundred forty six people attended our interactive exhibit and attendees noted the exhibit's ability to educate them about the healing process while also validating and shedding light on the experiences of sexual violence survivors. Our 